\title{
Risk-Informed Decision Making: More Critical Today Than Ever Before
}

\author{
Crystal Watson and Lucia Mullen
}

Keywords: Epidemic management/response, Risk assessment, Risk management, COVID-19, Decision making

$\mathrm{D}$ ECISION MAKING under uncertainty can be paralyzing for any leader trying to choose the best way forward. In the COVID-19 pandemic, uncertainty is deep; while we learn more every day, information gaps-including those related to viral transmission dynamics, the human immune response, the effectiveness of public health interventions like social distancing, and the future trajectory of COVID19 spread - continue to be barriers to leaders making evidence-based decisions about protective actions.

Decision making, especially during a pandemic, requires exceptional leadership that prioritizes rigorous approaches to producing high-quality data and then turning that evidence into action. Due to political pressure or attempts to calm the public, leaders may, instead, react quickly without considering the full impact of their choices. However, during pandemics and other emergencies, rational, evidence-based decision making is even more critical, especially when crucial decisions must be made despite gaps in information.

Risk assessment is an important element of evidencebased decision making, especially in an environment of deep uncertainty. It provides a way to structure analysis, interrogate possibilities, and reduce confounding human biases. In an effort to support decision makers during the COVID-19 pandemic, the Johns Hopkins Center for Health Security has collaborated with international organizations, national policymakers, and the private sector to provide tools to guide risk-informed, evidence-based decisions. Among these collaborations, the Center worked with the World Health Organization to develop decision trees and specific risk assessments for mass gatherings in the context of COVID-19. The Center has also provided ex- pertise to the United Nations to help their leadership make risk-informed decisions on which critical meetings should occur or be postponed, modified, or cancelled in context of this ever-changing pandemic. As the COVID-19 pandemic progresses, countries are beginning to lift strict social distancing measures and reopen their economies. For policymakers and private sector leaders, the Center has created risk assessment tools and guidance on how to reopen safely with a risk-informed approach.

This special section provides a broad definition of risk assessment and includes articles that describe different applications of risk analysis and risk management approaches. This is intentional. Risk assessment is versatile and can be used to anticipate and plan for future scenarios. It can also be used during an emergency to understand human health risks and interrogate response options and their anticipated impacts. Likewise, implementation of risk management approaches varies depending on the context. Approaches can be formal, like Enterprise Risk Management, which attempts to quantify both risk and the management steps employed to reduce risk, or they can be more informal, such as a small group of experts reviewing risks and weighing options for action.

The articles included in this special section address risks associated with events ranging from natural disasters to human-caused catastrophes. They include descriptions of risk management by a variety of actors in government, nongovernmental organizations, and the private sector engaged at the very local to the global level.

What these articles have in common is the systematic evaluation of the likelihood and consequences of events we

Crystal Watson, DrPH, MPH, is a Senior Scholar; and Lucia Mullen, MPH, is an Analyst; both are at the Johns Hopkins Center for Health Security, Baltimore, Maryland. 
fear and the processes to manage and reduce risk. They underline how risk management is a valuable tool for all stakeholders to use in their response to emergencies and other health events.

Although these articles were submitted prior to the current pandemic and do not address COVID-19 directly, the work described by the authors can inform our current response and help us anticipate future crises. The risk assessment and management approaches in these pages provide evidence that risk-informed decision making can and should be used to prevent, prepare for, and respond to health security threats. 